\title{
Utilización de aplicativo Remind como herramienta de apoyo en la enseñanza de investigación básica
}

\section{The use of Remind app as a support tool in basic research teaching}

\author{
Juan P. Matzumura-Kasano ${ }^{1, a}$, Hugo F. Gutiérrez-Crespo ${ }^{1, b}$, Raúl Alberto Ruiz-Arias ${ }^{2, c}$, Luis Huamán-Carhuas ${ }^{3, d}$ \\ ${ }^{1}$ Universidad Nacional Mayor De San Marcos. Lima, Perú. \\ ${ }^{2}$ Universidad Nacional Federico Villarreal. Lima, Perú. \\ ${ }^{3}$ Universidad Peruana Cayetano Heredia. Lima, Perú. \\ ${ }^{a}$ Médico gineco-obstetra, ORCID: http://orcid.org/0000-0003-0231-0187

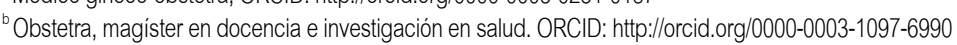 \\ ${ }^{\circ}$ Estadistico. ORCID: http://orcid.org/0000-0002 - 8877-6158 \\ ${ }^{d}$ Enfermero, magister en docencia e investigación en salud. ORCID: http://orcid.org/0000-0002-9463-6194
}

An Fac med. 2021;82(3):220-4. / DOI: https://doi.org/10.15381/anales.v82i3.20470

\section{Correspondencia:}

Hugo F. Gutiérrez Crespo

hgutierrezc@gmail.com

Recibido: 26 de mayo 2021

Aprobado: 4 de agosto 2021

Publicación en línea: 8 de septiembre

2021

Conflictos de interés: Los autores declaran no tener conflictos de interés.

Fuente de financiamiento:

Autofinanciado

Citar como: Matzumura-Kasano J, Gutiérrez-Crespo H, Ruiz-Arias A, Huamán-Carhuas L. Utilización de aplicativo Remind como herramienta de apoyo en la enseñanza de investigación básica. An Fac med. 2021;82(3):220-4. DOI: https://doi. org/10.15381/anales.v82i3.20470

\section{Resumen}

Objetivo. El estudio tuvo como objetivo determinar la relación entre la utilización del aplicativo Remind como herramienta de apoyo a la enseñanza y el rendimiento académico en un curso de investigación en estudiantes de medicina en 2020. Métodos. Investigación correlacional de corte transversal. Participaron estudiantes de medicina del curso de investigación básica de una universidad privada. Se aplicó a una muestra no probabilística de 96 estudiantes y se utilizó un cuestionario de tres dimensiones y 24 preguntas mediante una escala de Likert, con una confiabilidad de 0,94. Se emplearon estadísticos descriptivos y análisis bivariado. Resultados. La edad promedio fue de $23,2 \pm 5,9$ años, $68,8 \%$ fueron mujeres. 96,9\% utilizó el aplicativo por primera vez y 72,9\% lo considera seguro. Dimensión académica: $85 \%$ estuvo de acuerdo con la herramienta, porque permite enviar mensajes recordatorios de próximas clases. Dimensión aprendizaje: $79 \%$ aprendió a gestionar mejor sus tiempos y $77 \%$ lo considera una forma valiosa de aprendizaje. Dimensión personal: $71 \%$ estuvo de acuerdo con su uso y $69 \%$ lo recomienda en otros cursos. 85,4\% fueron del percentil favorable. Los estudiantes que usaron la herramienta presentaron mejores calificaciones. Conclusión. La aplicación tuvo aceptación favorable, facilitó el aprendizaje y fue positiva en las calificaciones.

Palabras clave: Tecnología de la Información y la Comunicación; Apoyo Educativo; Enseñanza; Investigación (fuente: DeCS - BIREME)

\section{Abstract}

Objective. The main goal of this study was to determinate the relationship between the use of Remind app as a support tool and the academic performance in teaching a research course to medical students in 2020. Methods. Correlational and cross-sectional research with medical students from a private university from the basic research course. This was applied to a non-probabilistic sample made of 96 participants. A 3 dimensioned and 24 questions questionnaire was used in the Likert scale, and contains (0.94) reability. Descriptive statistics and bivariate analysis were used. Results. The average age was $23.2 \pm 5.9$ years old, $68.8 \%$ were female. $96.9 \%$ used the app for the first time and $72.9 \%$ considered Remind as a safe app. Academic dimension: $85 \%$ agreed with the app as it allows to send reminders of the upcoming classes. Learning dimension: $79 \%$ learned to manage their time in a better way and $77 \%$ considered it a valuable form of learning. Personal dimension: $71 \%$ agreed in using it and $69 \%$ recommends using it in other courses. $85.4 \%$ are the favorable percentile. The students that used the app, had better grades. Conclusion. The app is favorably accepted; it facilitates the learning process and is positive to students' grades.

Keywords: Information Technology; Educational Support; Teaching; Research (source: MeSH NLM) 


\section{INTRODUCCIÓN}

En un contexto donde el modelo educativo por competencias se utiliza cada vez más, las universidades deberían buscar estrategias atractivas donde predomine la interacción profesor-estudiante ${ }^{(1,2)}$, así como tecnologías educativas y actualización de planes curriculares para desarrollar las competencias profesionales. Las aplicaciones móviles son herramientas utilizadas para el aprendizaje asincrónico. Se calculan más de 100000 aplicaciones vigentes dirigidas a la educación (3) algunas rediseñando actividades. Estas tecnologías continúan incrementándose sin pausa. Pueden ser usadas por estudiantes y profesores, generando también comprensión e incremento del rendimiento académico. Su integración puede redefinir las experiencias de aprendizaje y mejorar la calidad de la enseñanza ${ }^{(4)}$.

Los estudiantes utilizan la comunicación electrónica tradicional, siendo la mensajería de texto la preferida por la actual generación. Se calcula que $75 \%$ de los adolescentes la usan en su entorno familiar ${ }^{(5)}$. La aplicación gratuita Remind fue desarrollada en 2011 por Brett y David Kopf para dispositivos iOS y Android y para computadoras, desde la página oficial de la aplicación; está disponible en inglés, español, francés, alemán y chino mandarín. El profesor se comunica con los estudiantes enviando mensajes sobre los progresos, evaluaciones o mensajes recordatorios, documentos, presentaciones y archivos, manteniendo la privacidad del usuario. Es como el WhatsApp de los profesores, que permite construir y reforzar las comunicaciones dentro de un curso. Henderson y Mapp describieron su experiencia como una herramienta para la motivación, retroalimentación y mensajes motivadores, haciendo cumplir las tareas a los estudiantes con actividades fuera de clase ${ }^{(6,7)}$. El objetivo de esta investigación fue determinar la relación entre la utilización del aplicativo $R e$ mind, como herramienta de apoyo, y el rendimiento académico en la enseñanza de un curso de investigación básica en estudiantes de medicina durante el año 2020.

\section{MÉTODOS}

Se realizó una investigación con enfoque cuantitativo, correlacional y de cor- te transversal; la población de estudio estuvo conformada por estudiantes de medicina de una universidad de Lima, matriculados en el curso de investigación básica durante el año 2020. Se excluyó a participantes con una inasistencia mayor al 30\% y se empleó una muestra no probabilística por conveniencia.

El instrumento utilizado se elaboró según criterios de Hooper, Mora, Valerio ${ }^{(8)}$ y Heidari Tabrizi, Onvani ${ }^{(9)}$, conformado por 24 preguntas mediante una escala de Likert; contiene 3 dimensiones; académica, aprendizaje, personal. Tiene una confiabilidad de 0,94 según coeficiente Alfa de Cronbach. La puntuación se determinó mediante percentiles; no favorable (valor mínimo, percentil 39), regularmente favorable (percentil 40, percentil 56) y favorable (percentil 57, valor máximo). Asimismo, la medición de la variable rendimiento académico se realizó mediante el registro de calificaciones del curso.

La implementación del aplicativo Remind se realizó mediante actividades de motivación y registro del curso mediante el código @hc6dek. El análisis de datos se realizó mediante el programa SPSS v.23. Se emplearon estadísticos descriptivos, análisis bivariado para determinar la relación entre las variables. Se aplicaron los principios bioéticos dedicados a la investigación. Asimismo, se mantuvo la confidencialidad de los datos de los participantes durante la ejecución de la investigación.

\section{RESULTADOS}

La muestra final estuvo conformada por 96 participantes con una edad promedio de 23,2 años DE $\pm 5,9 ; 68,8 \%$ fueron mujeres. 96,9\% manifestó utilizar Remind por primera vez. $36,5 \%$ accedió mediante el sistema Android y $26 \%$ con iOS. $67,7 \%$ valoró la instalación con facilidad, 40,6\% refirió que Remind facilita estar al día sobre novedades o comunicados del curso, 33,3\% refirió que el aplicativo es fácil de utilizar y 55,2\% consideró como pertinentes los mensajes remitidos. Con respecto a la percepción de la utilidad educativa, 36,5\% manifestó que mejora la comunicación, $24 \%$ que ofrece una nueva forma de comunicación entre compañeros y $72,9 \%$ considera que la aplicación es segura.

Dimensión académica: 88\% estuvo de acuerdo con el aplicativo, ya que permitió enviar y recibir mensajes de todos los estudiantes, $85 \%$ respecto a que permitió enviar recordatorios de las próximas clases, $80 \%$ debido a que permitió enviar información sobre la próxima clase, tareas, prácticas o exámenes. Dimensión aprendizaje: 79\% manifestó que aprendieron el curso gestionando sus tiempos de la mejor forma y $77 \%$ consideró que fue una forma valiosa de aprendizaje y que fue un método diferente. Sin embargo, 32\% de los participantes manifestaron su indiferencia respecto a que el uso del aplicativo permita comprender mejor los puntos clave del curso y comprender contenidos teóricos. Dimensión personal: $71 \%$ estuvo de acuerdo con el uso de aplicativo, ya que mejoró sus habilidades tecnológicas y se sintieron satisfechos con su uso; 69\% recomendó su uso para otros cursos. Solo $10 \%$ manifestó que su aprendizaje mediante Remind causó ansiedad (Tabla 1).

Según la puntuación global: 2,1\% (2) se ubicó en el percentil no favorable, $12,5 \%$ (12) en regularmente favorable, $85,4 \%$ (82) en el percentil favorable y el promedio de calificaciones mediante el sistema vigesimal correspondió a 16,4 con una desviación estándar $\pm 1,3$. El analísis de correlación permitió observar que los estudiantes con mejor puntuación respecto al uso de Remind presentaron mejores calificaciones (rho=0,325) (Figura 1).

\section{DISCUSIÓN}

La utilización de aplicaciones en la enseñanza universitaria es una actividad común, debido a las demandas de comunicación entre estudiantes y profesores, generando un sentido de urgencia por obtener información de tipo académico o social. Las aplicaciones simplifican la organización, ayudan a responder preguntas de manera oportuna y ofrecen una fase de acompañamiento a los estudiantes en tiempo real ${ }^{(10,11)}$. Aunque algunos autores 


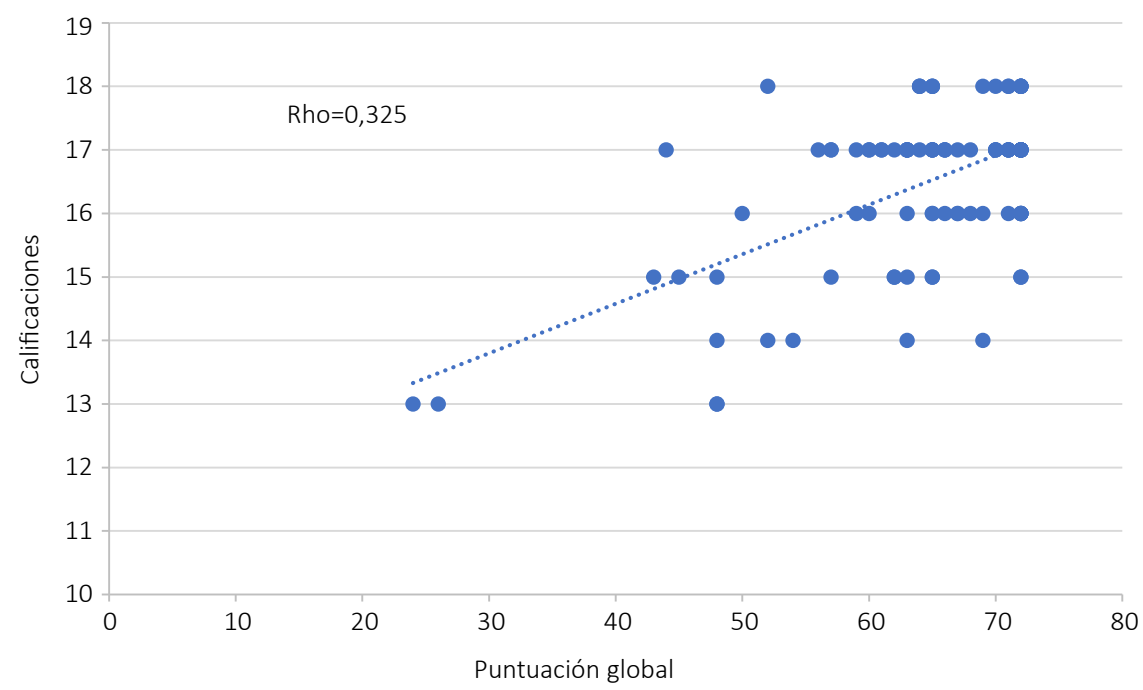

Figura 1. Análisis de correlación entre la utilización del Remind y calificaciones (rho de Spearman) en estudiantes de medicina del curso de investigación básica de una universidad privada de Lima, 2020.

favorecen el uso de estas aplicaciones, existen estudios que cuestionan su impacto y relevancia en el aprendizaje ${ }^{(1)}$.

Cabe indicar que el uso de Remind fue voluntario y no formó parte de la evaluación del curso; sin embargo, se utilizó para fomentar la comunicación de forma práctica y como un sistema recordatorio sobre las fechas importantes del desarrollo del curso como lo describen otras experiencias de similares características ${ }^{(12,13)}$. Se eligió Remind debido a las ventajas que ofrece sobre otras aplicaciones como WhatsApp o mensajería de texto. Remind no solicita al usuario información personal y permite que la comunicación siga siendo segura, instantánea, manteniendo la privacidad del participante ${ }^{(14)}$.

Las aplicaciones móviles tienen diversas características, como interfases individualizadas, acceso a información en tiempo real, comunicación instantánea y comentarios como los descritos por la mayoría de los participantes del presente estudio. Estas características han demostrado que mejoran el aprendizaje autodirigido y por indagación (15-17). Sin embargo, debemos señalar que usar aplicaciones móviles no es suficiente para obtener efectos positivos en el proceso de aprendizaje. Por ello, se plantea estrategias de instrucción para facilitar el aprendizaje efectivo con ayuda de la tecnología. Las investigaciones deben bus- car la integración de aplicaciones móviles con estrategias de instrucción y combinarlas de manera ingeniosa para resolver desafíos pedagógicos. ${ }^{(18-20)}$.

Abu Z. y Abdul R. reportaron que los mensajes de texto facilitan el aprendizaje y que pueden ser utilizados por los profesores como un método de evaluación flexible dentro del proceso de enseñanza y aprendizaje ${ }^{(21)}$. Además, que el servicio de envío de mensajes se realiza en forma instantánea con facilidad y permite ahorrar tiempo en la resolución de problemas, hacer que los estudiantes se sientan más vinculados con las actividades del curso y son útiles para estudiantes insatisfechos y con baja autoestima ${ }^{(22,23)}$.

Los resultados de nuestro estudio permiten reafirmar que Remind ofrece nuevas formas de aprendizaje y comprender los diversos contenidos que se imparten durante el desarrollo de curso, como lo describe Chiou Moh, en una investigación con estudiantes de una universidad de Taiwán; donde se evidenció comentarios positivos sobre el uso de teléfonos móviles y generaron mayores oportunidades de interacción entre los estudiantes, mejorando el desempeño durante los diversos cursos ${ }^{(24)}$. Del mismo modo, Kuznekoff y col. estudiaron el impacto del contenido del mensaje y su frecuencia durante el aprendizaje y evidenciaron que enviar y responder mensajes relacionados con el contenido de clases mejora las calificaciones de los estudiantes, ya que permite comprender y ordenar los diversos contenidos ${ }^{(25)}$. Similar hallazgo tuvo una investigación de Santos y col. sobre el uso de aplicaciones móviles en estudiantes. Esta concluye que es relevante en el proceso de enseñanza y aprendizaje, al proporcionar oportunidades para el intercambio de experiencias e información entre personas de diferentes realidades ${ }^{(26)}$. Por otra parte, el efecto puede ser contrario, si los mensajes se utilizan de manera extensiva, ocasionan distracción y no están relacionados con los contenidos del curso.

Sin embargo, otras investigaciones reportaron la presencia de correlación débil o negativa entre el uso de la tecnología como herramienta pedagógica y los resultados de aprendizaje. Si bien los aplicativos móviles ayudan en el proceso de aprendizaje, estos distraen y reducen el desarrollo de habilidades prácticas. Kraushaar y Novak sostienen que los estudiantes se distraen con el uso de aplicaciones no relacionadas con el curso, reduciendo su desempeño en los exámenes ${ }^{(27)}$. Mientras Wei y col. demostraron que los estudiantes que hacen uso de mensajes de texto, necesitan más tiempo para comprender las clases debido a su capacidad limitada de procesamiento de información cuando escuchan las clases y envían mensajes de texto, lo que reduce su aprendizaje ${ }^{(28)}$.

Dentro de las limitaciones de nuestro estudio, los resultados no mostraron cambios en los resultados del aprendizaje, y estos pueden verse afectados por la percepción de los estudiantes sobre su desempeño. Asimismo, los resultados no ofrecen recomendaciones sobre la utilización del aplicativo Remind que permitan mejorar los resultados de enseñanza y aprendizaje. La investigación no ofrece resultados comparativos con la utilización de otras aplicaciones o redes sociales, y el tamaño de muestra para futuras investigaciones debería ser mayor para un mejor análisis inferencial.

En conclusión, la aplicación Remind tuvo aceptación favorable por los estudiantes, la calidad de su desarrollo y contenidos facilitó el proceso de aprendizaje 
Tabla 1. Descripción de respuestas, por dimensiones, sobre la utilización de Remind en estudiantes de medicina del curso de investigación básica de una universidad privada de Lima, 2020.

\begin{tabular}{|c|c|c|c|c|c|c|c|c|}
\hline \multirow{2}{*}{ Preguntas } & \multicolumn{2}{|c|}{ No favorable } & \multicolumn{2}{|c|}{$\begin{array}{l}\text { Regularmente } \\
\text { Favorable }\end{array}$} & \multicolumn{2}{|c|}{ Favorable } & \multicolumn{2}{|c|}{ Total } \\
\hline & $\mathbf{n}$ & $\%$ & $\mathbf{n}$ & $\%$ & $\mathbf{n}$ & $\%$ & $\mathbf{n}$ & $\%$ \\
\hline \multicolumn{9}{|l|}{ Dimensión Académica } \\
\hline $\begin{array}{l}\text { Permite enviar recordatorios de las proximas clases o actividades } \\
\text { importantes }\end{array}$ & 3 & 3,1 & 11 & 12 & 82 & 85 & 96 & 100 \\
\hline Permite enviar y recibir mensajes a toda a clase & 4 & 4,2 & 8 & 8,3 & 84 & 88 & 96 & 100 \\
\hline $\begin{array}{l}\text { Permite enviar información sobre la proxima clase, tareas, prácticas } \\
\text { o exámenes }\end{array}$ & 3 & 3,1 & 16 & 17 & 77 & 80 & 96 & 100 \\
\hline $\begin{array}{l}\text { Mantiene informados a los estudiantes que faltan a clases, } \\
\text { enviando mensajes de texto }\end{array}$ & 8 & 8,3 & 26 & 27 & 62 & 65 & 96 & 100 \\
\hline $\begin{array}{l}\text { Mantiene informados a los estudiantes que faltan enviando } \\
\text { mensajes sobre las tareas pendientes }\end{array}$ & 4 & 4,2 & 22 & 23 & 70 & 73 & 96 & 100 \\
\hline $\begin{array}{l}\text { Permite compartir enlaces a sitios importantes que pueden ser } \\
\text { útiles para los estudiantes }\end{array}$ & 6 & 6,3 & 20 & 21 & 70 & 73 & 96 & 100 \\
\hline $\begin{array}{l}\text { Ha posible responder las preguntas de los estudiantes sobre la } \\
\text { tarea, material de la clase }\end{array}$ & 2 & 2,1 & 27 & 28 & 67 & 70 & 96 & 100 \\
\hline Se puede compartir comentarios personalizados y grupales & 4 & 4,2 & 21 & 22 & 71 & 74 & 96 & 100 \\
\hline Se puede anunciar algo que se olvido durante el desarrollo del curso & 3 & 3,1 & 20 & 21 & 73 & 76 & 96 & 100 \\
\hline \multicolumn{9}{|l|}{ Dimensión Aprendizaje } \\
\hline Ayuda a comprender mejor los puntos clave del curso & 3 & 3,1 & 31 & 32 & 62 & 65 & 96 & 100 \\
\hline $\begin{array}{l}\text { Ofrece oportunidades más efectivas para comprender y aprender } \\
\text { contenidos }\end{array}$ & 3 & 3,1 & 31 & 32 & 62 & 65 & 96 & 100 \\
\hline Ofrece flexibilidad en el aprendizaje & 2 & 2,1 & 26 & 27 & 68 & 71 & 96 & 100 \\
\hline Permite tener control sobre el aprendizaje & 6 & 6,3 & 28 & 29 & 62 & 65 & 96 & 100 \\
\hline $\begin{array}{l}\text { Es una forma valiosa de aprendizaje, diferente de otros métodos } \\
\text { clásicos de aprendizaje }\end{array}$ & 2 & 2,1 & 20 & 21 & 74 & 77 & 96 & 100 \\
\hline Aprender el curso con Remind es agradable y divertido & 4 & 4,2 & 27 & 28 & 65 & 68 & 96 & 100 \\
\hline Aprendern el curso con Remind, se puede gestionar de una mejor manera & 4 & 4,2 & 16 & 17 & 76 & 79 & 96 & 100 \\
\hline Aprender el curso a través de Remind es interesante y novedoso & 3 & 3,1 & 24 & 25 & 69 & 72 & 96 & 100 \\
\hline \multicolumn{9}{|l|}{ Dimensión Personal } \\
\hline $\begin{array}{l}\text { Con el uso de Remind, mi relación con mis compañeros y profesor } \\
\text { es beneficiosa y productiva }\end{array}$ & 2 & 2,1 & 34 & 35 & 60 & 63 & 96 & 100 \\
\hline Mejora mis habilidades tecnológicas (competencias digitales) & 4 & 4,2 & 24 & 25 & 68 & 71 & 96 & 100 \\
\hline Aprender el curso a través de Remind me hace más competente & 4 & 4,2 & 32 & 33 & 60 & 63 & 96 & 100 \\
\hline $\begin{array}{l}\text { El aprendizaje del curso con el uso de Remind crea menos } \\
\text { ansiedad }\end{array}$ & 10 & 10 & 32 & 33 & 54 & 56 & 96 & 100 \\
\hline Me siento menos inhibido de trabajar en el curso & 8 & 8,3 & 30 & 31 & 58 & 60 & 96 & 100 \\
\hline Estoy satisfecho con la aplicación & 5 & 5,2 & 23 & 24 & 68 & 71 & 96 & 100 \\
\hline Recomiendo el uso de Remind en otros cursos & 8 & 8,3 & 22 & 23 & 66 & 69 & 96 & 100 \\
\hline
\end{tabular}

y tuvo una influencia positiva en el desempeño y calificaciones de los estudiantes. La utilización constante de Remind, produjo una integración de la comunicación formal e informal en el proceso de enseñanza y aprendizaje con resultados positivos. Asimismo, ofrece una propuesta innovadora que permite integrar la tecnología en diversos entornos educativos y en la enseñanza de otros cursos.

\section{AGRADECIMIENTOS}

Al Sr. Dante Antonioli por su colaboración en la encuesta a los participantes del estudio.

\section{REFERENCIAS BIBLIOGRÁFICAS}

1. Santos-Guevara B, Acuña López A. Gammification and Remind App: An applied experience in a professional competencies development Workshop. iJEP 2020;10(2): 32-44. DOI: 10.3991/ ijep.v10i2.11632

2. González C, Area M. Breaking the rules: Gammification of learning and educational materials. En: Proceedings of the $2^{\text {nd }}$ International Workshop 
on Interaction Design in Educational Environments, Vol. 1; 2013 Jul 5-5; Angers, Francia. Setúbal: SciTe Press; 2013. p. 47-53. DOI: 10.5220/0004600900470053

3. Xuan-Lam P, Thi-Huyen N, Wu-Yuin H, Gwo-Dong C. Effect of push notifications on learner engagement in a mobile learning app. En: 2016 IEEE 16 ${ }^{\text {th }}$ International Conference on Advanced Learning Technologies (ICALT); 2016 Ago 25-28; Austin, EE.UU. Texas: IEEE; 2016. p. 90-4. DOI: 10.1109/ ICALT.2016.50

4. Marshall J. Quality teaching: Seven apps that will change the way you teach in the English language arts classroom. Voices from the Middle. 2016; 23(4): 66-73.

5. Lenhart A. Teens, smartphones \& testing. Pew Research Internet Project. 2012.

6. Gonzalez A. Strategies to get started with blended learning. Voices from the Middle. 2014; 22(2): 34-8.

7. Henderson AT, Mapp KL. A new wave of evidence: The impact of school, family and community connections on student achievement. Austin: National Center for Family and Community Connections with Schools. 2002.

8. Mora F, Valerio C. Hooper C. Uso de la aplicación Remind como herramienta de apoyo para la docencia en linea. En: XVII Congreso Internacional Innovación y Tecnología en Educación a Distancia; 2017 Nov 15-17; San José, Costa Rica. Madrid: UNED; 2017. p. 1-15.

9. Heidari Tabrizi H, Onvani N. The impact of employing Telegram app on Iranian EFL beginners' vocabulary teaching and learning. Applied Research on English Language. 2018; 7(1): 1-18. DOI: 10.22108/ARE.2017.103310.1087

10. Sung Y-T, Chang K-E, Liu T-Ch. The effects of integrating mobile devices with teaching and learning on students' learning performance: A meta-analysis and research synthesis. Computers \& Education. 2016; 94: 252-75. DOI: 10.1016/j. compedu.2015.11.008

11. Juarez B. Using the Remind App in the Online Classroom [Internet]. Faculty Focus [Citado 10 febrero 2021]. 2014 Disponible en: https://www. facultyfocus.com/articles/teaching-with-technology-articles/using-remind-app-online-classroom/

12. Ujakpa MM, Heukelman D, Lazarus VK, Neiss $P$, Rukanda GD. Using WhatsApp to support communication in teaching and learning. En: IST Africa Week Conference, Gaborone, Botswana, 2018 May 9-11. Texas: IEEE; 2018: p. 1-6.

13. Roig-Vila R (Ed.). La docencia en la enseñanza superior. Nuevas aportaciones desde la investigación e innovación educativas. Barcelona, España: Octaedro Editorial. 2020: p. 782-93.

14. McCoy K. What is Remind \& Why Should I Care? The advantages of utilizing a one-directional text message application to support student learning in academia. En: G. Chamblee \& L. Langub (Eds.). Proceedings of Society for Information Technology \& Teacher Education International Conference [Internet]; Savannah, Georgia, 2016 Mar 21-25. Waynesville, Carolina del Norte: Association for the Advancement of Computing in Education (AACE); 2014, p. 29-32. [Citado 7 marzo 2021]. Disponible en: https://www.learntechlib.org/ primary/p/171645/.

15. Sung $\mathrm{Y}-\mathrm{T}$, Yang J-M, Lee $\mathrm{H}-\mathrm{Y}$. The effects of mobile-computer-supported collaborative learning: Meta-analysis and critical synthesis. Review of Educational Research. 2017; 87(4): 768-805. DOI: 10.3102/0034654317704307

16. Lai CH, Yang JC, Chen FC, Ho CW, Chan TW. Affordances of mobile technologies for experiential learning: The interplay of technology and pedagogical practices. Journal of Computer Assisted Learning. 2007; 23(4): 326-37. DOI: 10.1111/j.1365-2729.2007.00237.x

17. Nisbet K, Opp A. Effects of the Remind app on parent-teacher communication at a mixed-income middle school [Tesis de Maestria]. Minnesota, EE.UU.: St. Catherine University; 2017. 38 p.

18. Lan KY, Sung YT, Tan NC, Lin CP, Chang KE. Mobiledevice-supported problem-based computational estimation instruction for elementary school students. Educational Technology \& Society. 2010; 13(3): 55-69.

19. Ozcelik E, Acarturk C. Reducing the spatial distance between printed and online information sources by means of mobile technology enhances learning: Using 2D barcodes. Computers \& Education. 2011; 57(3): 2077-85. DOI: 10.1016/j. compedu.2011.05.019

20. Oberg A, Daniels P. Analysis of the effect a student-centred mobile learning instructional method has on language acquisition. Computer Assisted Language Learning. 2012; 26(2): 1-20. DOI: 10.1080/09588221.2011.649484

21. Abu A, Abdul MF. Using SMS quiz in teaching and learning. Campus-Wide Information Systems. 2012; 30(1): 63-72. DOI: 10.1108/10650741311288823

22. Tang $Y$, Hew KF. Using Twitter for education: Beneficial or simply a waste of time? Computers \& Education. 2017; 106: 97-118. DOI: 10.1016/j. compedu.2016.12.004

23. Lauricella S, Kay R. Exploring the use of text and instant messaging in higher education classrooms. Research in Learning Technology. 2013; 21: 1-7. DOI: 10.3402/rlt.v21i0.19061

24. Moh C. Students acceptance of using smartphone in a mobile learning context. Educational Research and Reviews. 2015; 10(14): 1937-42. DOI: 10.5897/ ERR2015.2229

25. Kuznekoff J, Munz S, Titsworth S. Mobile phones in the classroom: Examining the effects of texting, Twitter, and message content on student learning. Communication Education. 2015; 64(3): 344-65. DOI: 10.1080/03634523.2015.1038727

26. Santos T, Soares LG, Machado LD, Brito N, Palácio MA, Silva MR. Use of mobile applications in the teaching-learning process in nursing graduation. Rev baiana enferm. 2021; 35:e37136. DOI: 10.18471/rbe.v35.37136

27. Kraushaar J, Novak D. Examining the effects of student multitasking with laptops during the lecture. Journal of Information Systems Education. 2010; 21(2): 241-51.

28. Wei FY, Wang YK, Klausner M. Rethinking college students' self-regulation and sustained attention: Does text messaging during class influence cognitive learning? Communication Education. 2012; 61(3): 185-204. DOI: 10.1080/03634523.2012.672755 